\title{
A comparative study of different network simulation tools and experimentation platforms for underwater communication
}

Tejaswini R. Murgod, S. Meenakshi Sundaram

Department of Computer Science and Engineering, GSSSIETW, Mysuru, India

\begin{tabular}{l} 
Article Info \\
\hline Article history: \\
Received Oct 25, 2020 \\
Revised Jan 17, 2021 \\
Accepted Feb 16, 2021 \\
\hline
\end{tabular}

\section{Keywords:}

Experimentation platforms

Network simulation

Underwater sensor networks

\begin{abstract}
Study of computer networks and their performance parameters like delay, bandwidth utilization, throughput, latency, jittering, and packet loss. have gained significant importance in the recent times. Simulation studies have been preferred for these parameters in computer networks, which in a real time is a difficult task. A network consists of many networking devices as gateways, routers, bridges, wireless access points and hub connected to it. To implement any new protocol or algorithm in a network is costlier and time consuming. Recently lot of research is going on underwater wireless sensor networks (UWSNs). Conducting real time experiments for underwater applications are overpriced, so as an alternative solution for this, we can conduct simulation studies to reduce the cost and quicken the research activities.In this paper we explore the different experimentation platforms and simulation tools available that help the network architects to develop new protocols or do changes to the existing protocol in a productive manner. We classify the tools based on various parameters and provide guidelines for researchers to choose a suitable platform based on their requirements.
\end{abstract}

This is an open access article under the CC BY-SA license.

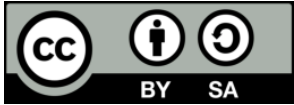

\section{Corresponding Author:}

Tejaswini R Murgod

Department of Computer Science and Engineering

GSSSIETW, KRS Rd, Metagalli, Mysuru, Karnataka 570016, India

Email: tejaswinirmurgod@gmail.com

\section{INTRODUCTION}

Simulation is an important and most widely used technology of modern times. The simulation is done to study the problematic and real-life objects on a computer. To design and develop new protocols simulations and field level experimentation are the two major steps to be carried out. Many commercial companies and researchers need simulation tools to test their algorithms before implementing in real hardware prototypes. The real life experiments guarantees that the solution is valid and achievable [1]. While conducting research for acoustic networks filed level deployments are more crucial, costlier and time consuming. By using simulation and emulation tools researchers can easily validate and debug underwater acoustic protocols in a cost effective way and can also shorten the turnaround time of the design [2].

Much of the research is being carried out till now for developing simulation tools and experimentation platforms for terrestrial sensor networks, but the channel characteristics of underwater acoustic sensor network (UWASN) are different when compared with terrestrial channels in terms of propagation delay, noise, penetration, path fading, and doppler effect. Therefore the platform developed for terrestrial networks cannot be used by the UWASN [3]. Many network simulators like NS2, NS3, OMNET++, J-SIM, and QualNet. are developed so that they can act as a testing tool in the implementation of specificfunctionality on a network. Since each simulator has its own unique features, it is an important task of researchers to identify the suitable simulator for their work prior to performance evaluation [4]. 
Different people from various areas like academician, researchers, developers, quality assurance can use these simulators to analyze and verify the performance of protocol designed. A simulator comprises of various technologies and protocols that helps researchers to construct complex network topologies using various types of nodes such as end-hosts, hubs, network bridges, routers, optical link-layer devices, and mobile units [5]. The aim of this survey is to recommenda wireless network simulator that provides a good balance between features like efficiency, extendibility, accuracy and easiness of use. By choosing such a simulator it allows the researchers to concentrate on their research rather than the simulator. This survey contains information about the challenges faced in underwater communication. Section 2 describes the different experimentation platforms and section 3 focuses on different simulation tools.

\section{EXPERIMENTATION PLATFORMS}

Researchers will face many challenges while designing and implementing a simulation tool. As the under water communication channel is continuouslychanging the challenge is to provide a selfconfigurable, robust platform that adapts to various environmental changes. Easily configurable, flexible, time synchronized, efficient, reliable and channel accurate testbed has to be developed that allows researchers to configure hardware or software parameters for testing different algorithms. The following criteria must be considered before selecting any simulation tool.

- Accuracy and efficiency: Real time distributed network applications requires time synchronization as their basic requirement, so the tools used for simulating the distributed network should ensure that the events occur correctly in timely bases. Before selecting the tool the researchers must go through the architecture of the tool, energy utilization, memory usage, power consumption and propagation speed of the network in order to capture an efficient and accurate behavior of the real underwater acoustic channel [6].

- Cross layer design support: The communication channel of underwater acoustic network is highly dynamic in which results in larger communication delay. To develop a protocol for the dynamic network, the simulation tools must allow researcher to exchange the cross layer information. The architecture of the tool used must be flexible enough to allow the researchers to easily configure the hardware and software parameters [7].

- Faultless transition support: Researchers do not consider the underwaterhardware while doing the initial simulation in order to eliminate the common errors in the protocol design. The same functionalities are then emulated on real acoustic network or sensor network in order to debug the errors in the underlying hardware, and then the field level testing of the protocol is done. This type of application requires faultless transition support in various development phases without tampering the information exchange.

- Modem support: Testbeds and simulation tools must support commercial off the shelf modems and research-oriented underwater acoustic modems. To handle the environmental changes and to support cross layer design reconfigurable modems are needed, which allows researchers to change the modem configuration in a flexible manner [8].

The Table 1 lists the different experimentation platforms for under water wireless sensor networks.

Table 1. Different experimentation platforms for UWSN

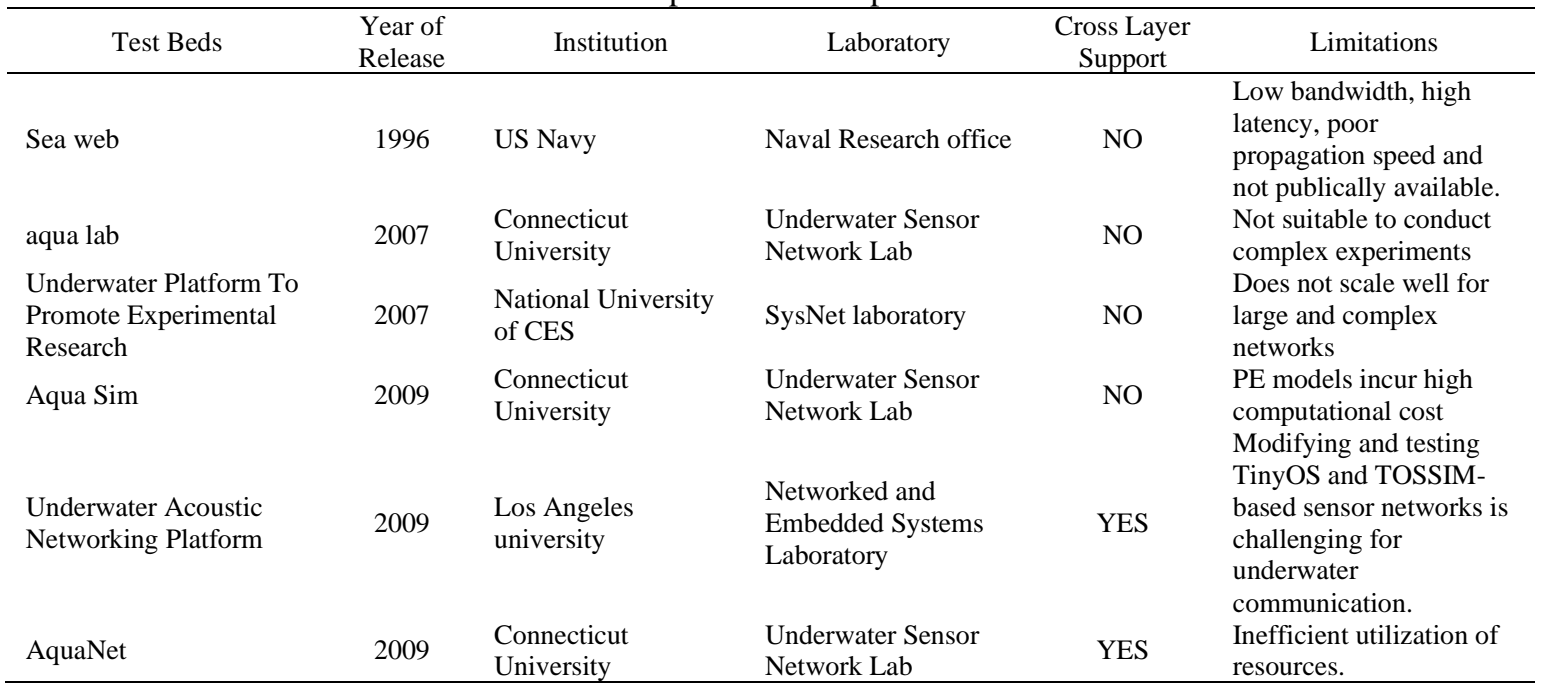


Table 1. Different experimentation platforms for UWSN (Continue)

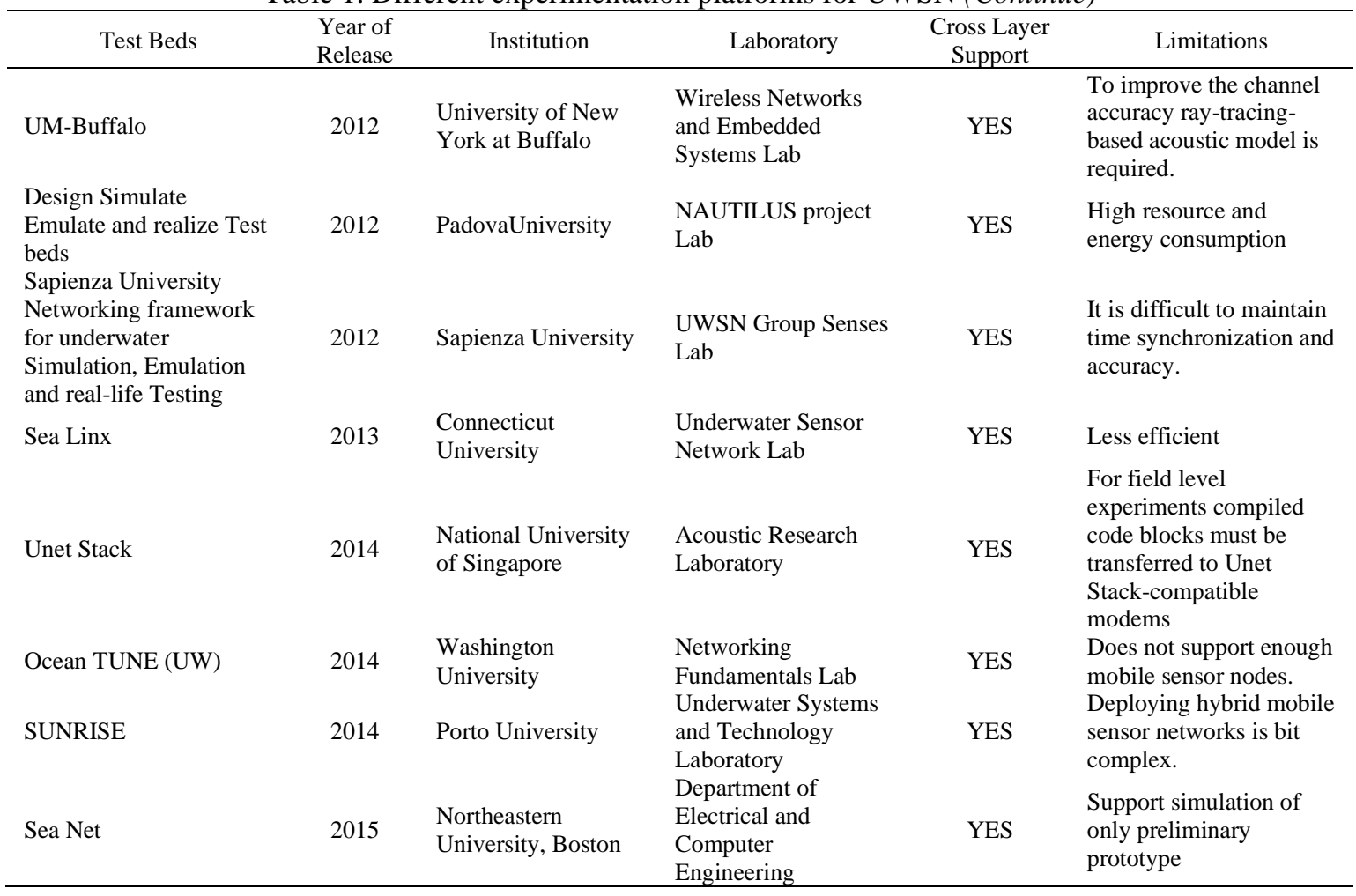

- Aqua lab: It contains a set of API's that provide the abstract view of the operations to the users, which help them to develop their own application without the knowledge of the underlying physical layer. Underwater speakers and hydrophones are used as transducers and sound mixer is used to introduce the acoustic signals into the channel. Server controls the operation of all the components. C compliant library functions are used at lower and higher level. Lower level functions take care of setting the baud rate, timeout for data requests, opening and closing the port for communication, higher level functions are used to provide ease of use to the users. Aqua lab is not suitable to conduct complex experiments but beginners can use this to their own environment at low cost [9].

- Underwater platform to promote experimental research (UPPPER): It is a flexible and low-cost underwater platform. It makes use of COTS components to provide cost effective platform. To support stack integration Shim Layer is used. Shim Layer provides half duplex packet interface to the modems. Shim Layer can be extended for remote access through RPC interface. UPPER classifies the software components as implementation of Shim layer and GNU Radio based software modem. Shim layer provides interaction between external users and software modems by enabling flow control and rate control. Shim layer is implemented in three parts: RPC server, GNU radio interface and queue management. RPC server adds the header to the packets and forwards it to the transmit queue. The transmit queue provides flow control. If users transmit more packets than the capacity of the physical layer, then buffering is done at Shim layer and transmitted when physical layer is free. The modem consists of three sub parts as multiplexing logic, transmit and receive flow graphs [10].

- Aqua sim: It is a package of NS-2 which is independent of the other packages. Aqua Sim is organized into 4 folders

- uw_common: contains code for simulating underwater nodes and traffic

- uw_mac: contains code for acoustic channel and MAC protocols simulation

- uw_routing: contains code for simulating all routing protocols

- uw_tcl: contains octl scripts to validate Aqua sim [11]

- Underwater acoustic networking platform (UANT): To provide configurability at physical layer UANT use GNU radio signals and Tiny OS for MAC layer. Linux assigns an IP address for UANT. Transducers receive the packets through acoustic signals and pass it to the USRP which will perform sampling and convert the data to the specific format [12].

- Aqua net mate: Aqua net mate is a user-friendly simulator which is easily configurable, and expandable in real time. State machine design is used to imitate the real acoustic modems. It provides transparency so 
that at any time we can switch under layers between simulation mode and experimentation mode. To provide transparency, it makes uses of two components, virtual modem which emulates the real modem and Aqua Net driver referred as adapters which is used to provide interface with virtual modems. Virtual channel is used to pass all the packets sent by virtual modems. It adopts object oriented design where model channel, propagation, attenuation are all defined in base class. If any advancement needs to be done then the base class needs to be inherited and overridden with the specified behavior [13].

- Design simulate emulate and realize test beds (DESERT): DESERT provides multi modal communication functionalities to achieve high data rate for underwater acoustics and optical networks. It allows connection to WOSS (World Ocean Simulation System). The improved version of DESERT provides modules for simulating optical propagation. Background ambient light noise is considered to form the depth look up table. Optical communication is employed for efficient transfer of bulk data [14].

- Sapienza university networking framework for underwater simulation, emulation and real-life testing (SUNSET): It is based on NS-2 Simulator. Researchers can run tests without changing the external device code. It requires limited effort to evaluate their protocol solutions. It uses different acoustics channels models as ray tracing, WOSS Interface. SUNSET architecture is flexible enough to integrate any external device. Software API's are used to control the operations of the device. It can also be easily ported to small portable devices allowing the user to easily embed it in the modem. It uses acoustic communication to transfer the data packets to the static or remote devices connected in the network [15].

- SeaLinx: SeaLinx is multi-instance layered protocol stack architecture for underwater network. The protocol covers different layers such as MAC layer, network layer, transport layer and application layer. Each protocol is organized into separate processes thus allowing multiple protocols running at different layers. Communication can be established between any layers with the help of the core layer. When a message arrives at the network layer, the protocol checks whether it can handle it, if so it will accept the message else it simply discards it. SeaLinx provides flexibility for cross layer protocol [16].

- Ocean tune (Water com): Water com frame work provides a mechanism to integrate external simulators into an experimentation configuration. Optical modems are used which uses laser or LED's to encode data and photons gathered from sensitive detector are used to decode the data. The communication range is highly dependent on the brightness, water turbidity, detector sensitivity and intensity of the light source. Water com uses a transmission tube that contains underwater mirrors as transmitter and receivers and optical transmission hardware are placed above the water line to avoid water proofing and maintenance issues [17].

- SUNRISE: Sunrise implements software defined modem and communication stack which allows cross layer optimization, adaptive underwater communication protocols. It provides large scale testing infrastructure. It also reduces time to experiment by providing tools to support code generation, programmability, reprogramming underwater deployments [18].

- Software defined acoustic networking (SEANet): SEANet provides various set of functionalities at different layers which provides UAN devices to adapt to different application and system requirements. It provides a modular structured architecture that enables cross layer reconfiguration, platform independence, flexibility to design new protocol design and software portability. The limitations of commercial devices are overcome by using UAN device [19].

\section{NETWORK SIMULATION TOOLS}

Network simulators provide an integrated, versatile, easy-to-use GUI-based network designer tool to design and simulate a network. Different network simulation tools are available to simulate the real world network with different features. Here we compare the different network simulators.

\subsection{Network simulator (NS)}

NS2: Network simulator version 2 is a simple event-based simulation tool used for studying the dynamic nature of the network. NS2 provides simulation of wired and wireless network function protocols. NS2 uses OTcl to create and configure a network and uses C++ to run the simulation [20]. The latest version of NS2 is NS2.35 released in the year 2011.

NS 3: NS3 project started in the mid of 2006 and was funded by University of Washington, INRIA, Sophia Antipolis, Georgia Tech University. It has been developed to provide an open, extensible network simulation platform, for networking research and education. NS 3 is designed as a set of libraries and it can be combined with other external software libraries also. Some of the features of NS3 are that, it provides scalability, provides cross layer functionalities. Small amount of information is attached to each packet that 
allows to report about event occurrence across non-contiguous layers and supports real world integration. The latest version is NS -3.29 and was released on 4 September 2018 [21] with the following new features:

- $\quad$ To provides a 3GPP reference model for HTTP/1.1

- $\quad$ For traffic control a priority queue is used

- $\quad$ Provides support for TCP Proportional Rate Reduction (PRR) recovery algorithm

- Supports a position allocator algorithm that rejects positions that are defined in the scenario

OPNet: Opnet Network Simulator can be used to simulate any type of network. As it provides versatility and less power consumption it is widely used by many researchers. Some of the key features of OPNet are

- $\quad$ Provides support for analysis, building and execution of models

- Support hierarchical structure of modeling

- A detailed library is provided for different models [22]

OMNET++: OMNET ++ provides modular architecture which enables the simulation kernel to be easily embedded into any applications. A component-based framework and library is provided which makes building of a network simulators an easier task. It offers eclipse-based IDE and a graphical user defined environment. OMNET ++ does not provide the components directly for simulation but instead it just provides the basic tools and machinery required to write such simulators. OMNET++ 5.4.1 was released on June 29, 2018 [23].

QualNet: It is a cost-effective method of developing, deploying and managing network centric systems, which enables users to evaluate the basic behavior of a network. An environment is provided for designing protocols, creating and animating network scenarios and analyzing their performance. It also enables users to design and optimize new protocol models, design large wired/wireless networks and also to analyze the performance of the network. The key features of QualNet are:

- It supports real time speed for network emulators. Faster speed enables developers to run network traffic parameters at shorter time

- $\quad$ Can run more than 1000 of nodes by using latest hardware and parallel computing techniques. It can run on multi-core, multi-processor system

- $\quad$ It can run on various platforms and provide portability

- Provides extensibility by allowing connecting to other hardware and software applications such as OTB, real networks to enhance the value of network model

The latest version of QualNet is QualNet 7.4. This new version provides advanced wi-fi modeling capabilities, improved database, and cyber attack analysis. Updated model includes 802.11p PHY and MAC Protocols known as WAVE [24].

J-Sim: It is an open source, component-based compositional network simulation environment developed entirely in Java. It provides a platform neutral, extensible and reusable environment. It also allows to integrate with different scripting languages like perl and python [25]. The latest J-Sim version 2.18 was released on 5 September 2018. Some of the features of J-Sim are:

- $\quad$ Provides autonomous and loosely coupled components

- $\quad$ For real time simulations it provides dynamic thread execution framework

- It provides dual language environment where java is used to create components and a scripting language is used to integrate components at run time

- Generic interface classes are defined and implemented for trace-driven simulation

NetSim: NetSim comes in three versions Standard, Pro and Academic. Standard version is used for research and project work, Pro version is used to fulfill the needs of industry and defense, Academic version is used for teaching and conduction lab experiments. The main difference between standard and academic version is the availability of protocol source code, and packet tracing facility. The latest version is NetSim v11.0 was released in August 2018 with the following added features

- It provides support to SDN based IOTs, WSN, LTE and VANETS

- $\quad$ Switching port and links now support 10G, 40G and 100G

- $\quad$ Provides real time interaction with simulation with windows equivalent commands

- $\quad$ GMSK modulation scheme is added at PHY Layer

- $\quad$ Fast configuration option for simulating networks with large number of devices

- $\quad$ GUI is enhanced by providing Tool tip help, display of error messages, copy paste options for device and links

Matlab: MATLAB (matrix laboratory) is amulti-paradigmnumerical computingenvironment andproprietary programming languagedeveloped byMathWorks. The latest release of Matlab is R2018b (version 9.5) was released in September 2018 with the following features

- Live Editor: Organize live scripts using additional subheading styles

- Drag and drop visual components to lay out the design of your app

- Create a virtual representation of a real-world system that includes software and hardware. 
The Table 2 gives the comparison of different network simulators in various dimensions.

Table 2. Comparisons of different network simulators

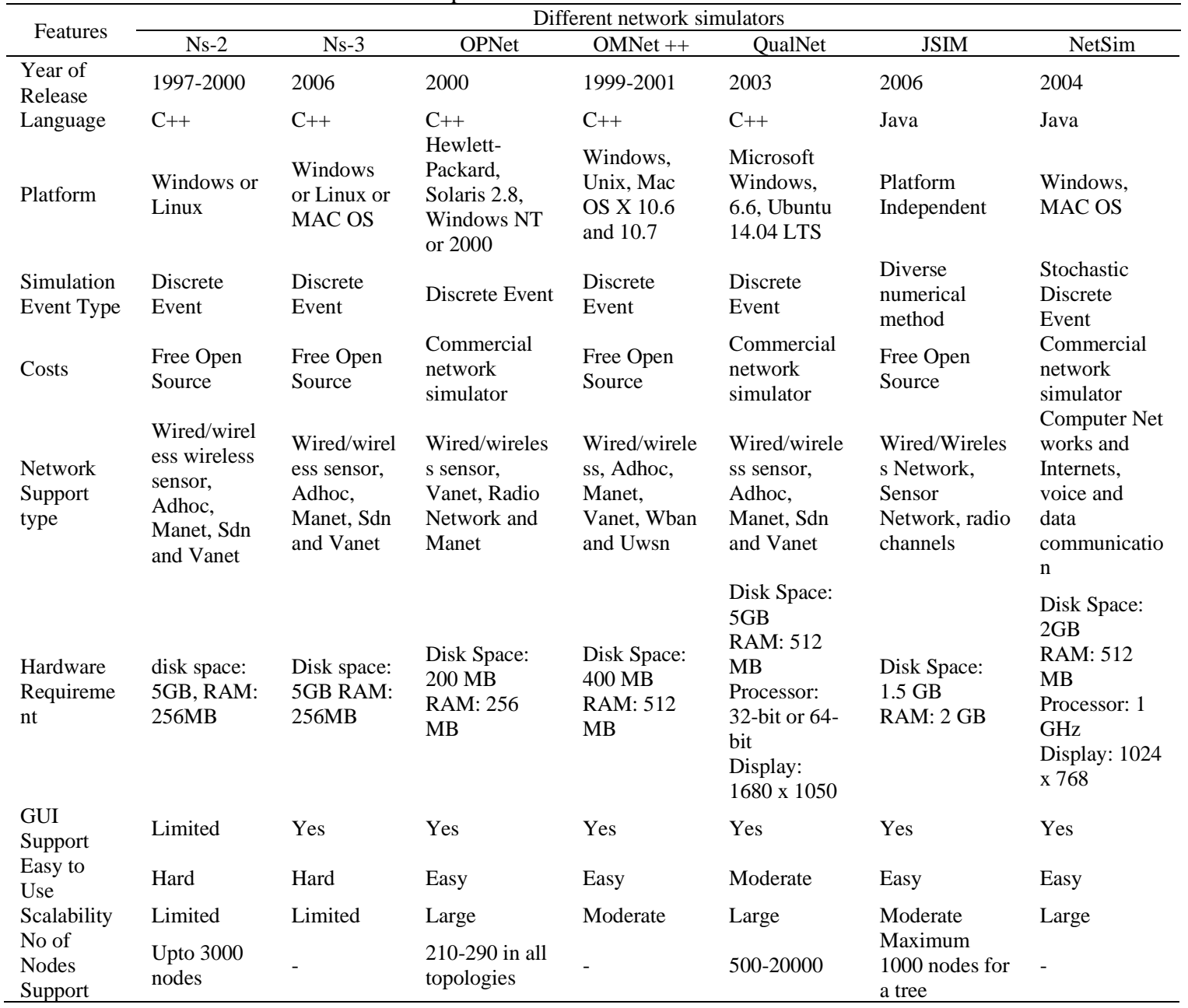

\section{CONCLUSION}

In this paper we presented a survey on underwater simulation tools and experimental platforms. The architecture features, and limitations of each platform are discussed separately. We further provided a comparative study among the selected platforms. Based on the comparative study, we present some advice for researchers on choosing suitable platforms for underwater communication. The comprehensive survey of underwater communication based on the usage of tools in the papers and based on user rating on ease of use of the tool. Hence we conclude that QualNet, Matlab, NS2 are the best suitable for underwater communication.

\section{REFERENCES}

[1] Luo, Hanjiang, Kaishun Wu, Rukhsana Ruby, Feng Hong, ZhongwenGuo, and Lionel M. Ni. "Simulation and experimentation platforms for underwater acoustic sensor networks: Advancements and challenges," ACM Computing Surveys, vol. 50, no. 2, p. 28:3, 2017.

[2] Burtovaya, D., A. Demin, M. Demeshko, Alexander Moiseev, and A. Kudryashova. "Simulation of the communication system between an AUV group and a surface station," In Journal of Physics: Conference Series, vol. 803, no. 1, p. 012027, 2017.

[3] Pan, Jianli, and Raj Jain, "A survey of network simulation tools: Current status and future developments," 2008.

[4] Kang, SeokYoon, MontherAldwairi, and Ki-Il Kim, "A survey on network simulators in three-dimensional wireless ad hoc and sensor networks," International Journal of Distributed Sensor Networks, vol. 12, no. 10, pp. 1-10, 2016. 
[5] S. Mehta, N. Ullah, M. H. Kabir, M. N. Sultana and K. S. Kwak, "A Case Study of Networks Simulation Tools for Wireless Networks," 2009 Third Asia International Conference on Modelling\& Simulation, Bali, pp. 661-666, 2009.

[6] Dhurandher, Sanjay K., Mohammad S. Obaidat, and Megha Gupta, "An acoustic communication based AQUAGLOMO simulator for underwater networks," Human-centric Computing and Information Sciences, vol. 2, no. 3, pp. 1-14, 2012.

[7] Martinez, Francisco J., Chai KeongToh, Juan-Carlos Cano, Carlos T. Calafate, and Pietro Manzoni, "A survey and comparative study of simulators for vehicular ad hoc networks (VANETs)," Wireless Communications and Mobile Computing, vol.11, no. 7, pp. 813-828, 2011.

[8] J. Rice et al., "Evolution of Seaweb underwater acoustic networking," OCEANS 2000 MTS/IEEE Conference and Exhibition. Conference Proceedings (Cat. No.00CH37158), Providence, RI, USA, vol. 3, pp. 2007-2017, 2000.

[9] Peng, Zheng, Jun-Hong Cui, Bing Wang, Keenan Ball, and Lee Freitag, "An underwater network testbed: design, implementation and measurement," In Proceedings of the second workshop on Underwater networks, ACM, pp. 65-72, 2007.

[10] Bin Abbas, Waqas, Niaz Ahmed, ChaudhryUsama, and Affan A. Syed, "Design and evaluation of a low-cost, DIYinspired, underwater platform to promote experimental research in UWSN," Ad Hoc, vol. 34, pp. 239-251, Nov 2015.

[11] P. Xie et al., "Aqua-Sim: An NS-2 based simulator for underwater sensor networks," OCEANS 2009, Biloxi, MS, pp. 1-7, 2009.

[12] Torres, Dustin, Jonathan Friedman, Thomas Schmid, Mani B. Srivastava, Youngtae Noh, and Mario Gerla, "Software-defined underwater acoustic networking platform and its applications," Ad Hoc Networks, vol. 34, pp. 252-264, Nov 2015.

[13] Y. Zhu et al., "Aqua-Net Mate: A real-time virtual channel/modem simulator for Aqua-Net," 2013 MTS/IEEE OCEANS - Bergen, Bergen, pp. 1-6, 2013.

[14] F. Campagnaro et al., "The DESERT underwater framework v2: Improved capabilities and extension tools," 2016 IEEE Third Underwater Communications and Networking Conference (UComms), Lerici, pp. 1-5, 2016.

[15] Petrioli, Chiara, and Roberto Petroccia, "SUNSET version 2.0: Simulation, emulation and real-life testing of underwater wireless sensor networks," WUWNet '13: Proceedings of the Eighth ACM International Conference on Underwater Networks and Systemsce, no. 43, pp. 1-8, 2013.

[16] S. Hurst, X. Xie, S. Ashrafi, S. Roy and P. Arabshahi, "Puget sound underwater networking TestBed," 2014 Oceans - St. John's, St. John's, NL, pp. 1-5, 2014

[17] Goldrick, CiaránMc, Mark Matney, Enrique Segura, Youngtae Noh, and Mario Gerla, "Watercom: A multilevel, multipurpose underwater communications test platform," In Proceedings of the 10th International Conference on Underwater Networks \& Systems, no. 14, pp. 108, Oct 2015.

[18] R. Martins, J. B. de Sousa, R. Caldas, C. Petrioli and J. Potter, "SUNRISE project: Porto university testbed,"2014 Underwater Communications and Networking (UComms), Sestri Levante, pp. 1-5, 2014.

[19] Demirors, Emrecan, Bharatwaj G. Shankar, G. Enrico Santagati, and TommasoMelodia, "SEANet: A softwaredefined acoustic networking framework for reconfigurable underwater networking," In Proceedings of the 10th International Conference on Underwater Networks \& Systems, no. 11, pp. 1-8, Oct 2015.

[20] Issariyakul, Teerawat, and EkramHossain, "Introduction to Network Simulator 2 (NS2)," In Introduction to Network Simulator NS2, Springer, Boston, MA, pp. 1-18, 2008.

[21] GarimaBhardwaj, Sandhya K., RichaDolia, M. Abu-Samak, Shalendra Kumar, P. A. Alvi, "A Comparative Study on Optical Characteristics of InGaAsP QW Heterostructures of Type-I and Type-II Band Alignments," Bulletin of Electrical Engineering and Informatics, vol. 7, no. 1, pp. 35-41, March 2018.

[22] Amanjot Singh Toor, A. K. Jain, "A Survey on Wireless Network Simulators," Bulletin of Electrical Engineering and Informatics, vol. 6, no. 1, pp. 62-69, March 2017.

[23] A. Tamizharasi M. E., J. Jasmine Selvathai M. E., A. KaviPriya, Maarlin R., Harinetha M, "Energy Aware Heuristic approach for Cluster Head Selection in Wireless Sensor Networks," Bulletin of Electrical Engineering and Informatics, vol. 6, no. 1, pp. 70-75, March 2017.

[24] Fengmei Liang, Liyuan Zhang, Peng Sun, "Study on the Rough-set-based Clustering Algorithm for Sensor Networks," Bulletin of Electrical Engineering and Informatics, vol. 3, no. 2, pp. 77-90, June 2014.

[25] Shengjun Su, Shuozhong Wang, "A Simple Monitoring Network System of Wireless Sensor Network," Bulletin of Electrical Engineering and Informatics, vol. 1, no. 4, pp. 251-254, Dec 2012. 